\title{
A Comparison of Capillary Versus Venous N-Terminal Prohormone Brain Natriuretic Peptide
}

\author{
Vahid Mehrnoush ${ }^{1}$, Andrew Kochan ${ }^{2}$, Solmaz Ehteshami-Afshar ${ }^{3}$, Sean A Virani ${ }^{4}$, Nathaniel M Hawkins ${ }^{4 *}$ \\ and Mustafa Toma ${ }^{4}$ \\ ${ }^{1}$ Urology Department, Northern Ontario School of Medicine, Canada \\ ${ }^{2}$ Department of Internal Medicine, University of British Columbia, Canada \\ ${ }^{3}$ Department of Internal Medicine, Yale University School of Medicine, United States \\ ${ }^{4}$ Division of Cardiology, University of British Columbia, Canada
}

\begin{abstract}
Background: The natriuretic peptides B-type natriuretic peptide and N-terminal fragment B-type natriuretic peptide provide a relatively low-cost and accessible screening tool for heart failure. Point of care testing using finger prick capillary blood samples has been developed for BNP. We compared capillary versus venous N-terminal fragment B-type natriuretic peptide measurements using a commercially available point of care testing assay.
\end{abstract}

Methods: A cross-sectional prospective sub-analysis of a study screening 67 patients with stable chronic obstructive pulmonary disease for cardiovascular disease using N-terminal fragments of B-type natriuretic peptide was performed. Capillary and venous blood samples for each patient were analysed using a point of care testing N-terminal fragment B-type natriuretic peptide whole blood assay. Correlation between capillary and venous N-terminal fragment B-type natriuretic peptide levels was assessed. The probability of heart failure was classified based on age-stratified $\mathrm{N}$-terminal fragment B-type natriuretic peptide levels.

Results: Mean capillary versus venous N-terminal fragment B-type natriuretic peptide values were similar, 236 $\pm 530 \mathrm{pg} / \mathrm{mL}$ vs. $237 \pm 512(\mathrm{n}=67)$, with high linear correlation $(\mathrm{R} 2=0.96, \mathrm{P}<0.05)$. Capillary testing reclassified 5 of the 39 patients $(13 \%)$ designated low probability of HF by venous testing as having intermediate values, mainly in elderly patients. No patients designated as having a moderate or high probability of having venous testing were reclassified by capillary testing.

Conclusion: There is a good correlation between N-terminal fragment B-type natriuretic peptide levels in capillary and venous samples, which suggests there may be a role for point of care testing of N-terminal fragment B-type natriuretic peptide using capillary blood samples. These findings need to be confirmed in larger populations, including more patients with heart failure.

KEYWORDS: Heart failure; Natriuretic peptide; Point of care testing

ABBREVIATIONS: BNP: B-type Natriuretic Peptide; COPD: Chronic Obstructive Pulmonary Disease; HF: Heart Failure; LVSD: Left Ventricular Systolic Dysfunction; NT-proBNP: N-terminal Fragment B-Type Natriuretic Peptide; POCT: Point of Care Testing

Quick Response Code:

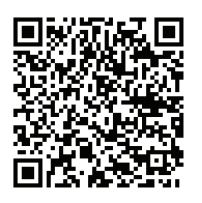

Address for correspondence: Nathaniel M Hawkins, Department of Medicine, Division of Cardiology, University of British Columbia, BC Centre for Improved Cardiovascular Health, Vancouver, Canada

Received: October 07, $2021 \quad$ Published: October 22, 2021

How to cite this article: Mehrnoush V, Kochan A, Afshar SA, Virani SA, Hawkins NM, Toma M. A Comparison of Capillary Versus Venous N-Terminal Prohormone Brain Natriuretic Peptide. 2021- 3(5) OAJBS.ID.000339. DOI: 10.38125/OAJBS.000339 


\section{INTRODUCTION}

Heart failure (HF) is a worldwide epidemic that causes frequent hospitalizations and high health-care costs. Prognosis remains poor despite numerous medications and devices which improve symptoms and prolong survival [1]. Therapies halt or even reverse left ventricular systolic dysfunction (LVSD). This process, termed left ventricular remodeling, occurs over years. Early detection and treatment of HF are therefore paramount [2,3]. Symptoms and signs alone exhibit low diagnostic accuracy for HF, which requires an objective demonstration of cardiac dysfunction. This is typically provided by imaging such as echocardiography, which presents barriers in terms of access and costs [4]. The natriuretic peptides (NP) B-type natriuretic peptide (BNP) and N-terminal fragment BNP (NT-proBNP) are released in response to ventricular stretch in patients with HF. High and low NP levels suggest a high and low probability of HF respectively, the latter having high negative predictive value. Natriuretic peptides provide a relatively low-cost and accessible method to screen patients for confirmatory imaging and to initiate therapy [1,4-6].

Natriuretic peptides were traditionally assayed in laboratories using venous blood samples. Development of Point of Care Testing (POCT) has enabled expedited measurement and management [4]. However, most POCT still requires venous blood and thus phlebotomy services, which limits availability $[7,8]$. Recently, several commercial BNP POCT assays have been tested on capillary 'fingerprick' samples with encouraging results [8-11]. Treatment with angiotensin receptor blocker-neprilysin inhibitors, which increase BNP levels, has increased demand for NT-proBNP compared to BNP testing $[12,13]$. We therefore aimed to compare capillary versus venous NT-proBNP measurements using a commercially available POCT assay based on the RAMP $®$ technology platform.

\section{MATERIALS AND METHODS}

\section{Design and Population}

The cross-sectional study was prospectively designed as a sub-analysis of a study screening ambulatory patients with stable chronic obstructive pulmonary disease (COPD) for cardiovascular disease using NT-proBNP from February 2017 to July 2018. Consecutive patients with stable COPD attending pulmonary clinics at Vancouver General Hospital and St. Paul's Hospital The inclusion criteria were: 1) clinical diagnosis of COPD with confirmatory spirometry; 2) stable respiratory state for at least 4 weeks, i.e. no recent hospitalization attributed to COPD, no respiratory tract infections requiring antibiotics or steroids, and unchanged doses of concomitant respiratory therapy. Patients with established heart failure were excluded.

\section{Assay, Sampling and Data Collection}

A POC NT-proBNP whole blood assay was supplied by Response Biomedical Corporation (RAMP® kit and Reader). Venous (1-2ml) and capillary (a few drops of finger-stick sample collected by a small pipette) blood samples were drawn from each patient. An aliquot $(75 \mu \mathrm{L})$ from each sample was mixed 10 times with the buffer solution and applied to a single-use disposable cartridge. Baseline demographics, medical history, medications, physical examination and clinical parameters of interest were recorded. All data was stored in an electronic case report form using REDCap.

\section{Statistical Analyses}

Baseline characteristics of patients are presented as means with standard deviations for continuous variables or by frequencies and percentages for categorical variables. Venous and capillary values are presented by means of a scatter plot with a correlation coefficient. The proportion of patients with low, medium or high NT-proBNP levels was calculated based on the recommended lower exclusionary threshold of $300 \mathrm{pg} / \mathrm{mL}$, and age specific upper cut-points from the International Collaborative of NT-proBNP dataset: $<50$ years $450 \mathrm{pg} / \mathrm{mL}$; $50-75$ years $450-900 \mathrm{pg} / \mathrm{mL} ;>75$ years $>900 \mathrm{pg} / \mathrm{mL}$ [14]. The proportion of patients misclassified using capillary compared to the gold standard of venous blood was calculated. Bland-Altman difference plots were constructed to assess the limits of agreement. The study was approved by the University of British Columbia Research Ethics Board. All analyses were performed using SPSS for Windows v21.0 (SPSS Inc., Chicago, Illinois).

\section{RESULTS}

\section{Baseline Characteristics}

Table 1: Baseline characteristics.

\begin{tabular}{|c|c|}
\hline Demographics & Mean \pm SD or Frequency $(\%)$ \\
\hline Male & $46(68.7 \%)$ \\
\hline Age & $71.5 \pm 10.0$ \\
\hline Body mass index (BMI) & $29.2 \pm 14.1$ \\
\hline Overweight $(25 \leq \mathrm{BMI}<30)$ & $20(29.9 \%)$ \\
\hline Obese $($ BMI $\geq 30$ ) & $24(35.8 \%)$ \\
\hline \multicolumn{2}{|c|}{$\begin{array}{c}\text { Chronic Obstructive Pulmonary Disease (GOLD } \\
\text { classification) }\end{array}$} \\
\hline I Mild & $2(3.0 \%)$ \\
\hline II Moderate & $37(55.2 \%)$ \\
\hline III Severe & $24(35.8 \%)$ \\
\hline IV Very severe & $1(1.5 \%)$ \\
\hline \multicolumn{2}{|c|}{ Cardiovascular Risk Factors } \\
\hline Smoking & $62(92.5 \%)$ \\
\hline Diabetes & $13(19.4 \%)$ \\
\hline Hypertension & $25(37.3 \%)$ \\
\hline Dyslipidaemia & $27(40.3 \%)$ \\
\hline \multicolumn{2}{|c|}{ Cardiovascular Disease } \\
\hline Atrial fibrillation & $7(10.4 \%)$ \\
\hline Angina & $12(17.9 \%)$ \\
\hline Coronary artery disease & $13(19.4 \%)$ \\
\hline Myocardial infarction & $7(10.4 \%)$ \\
\hline History of valve disease & $2(3.0 \%)$ \\
\hline Transient ischemic attack or stroke & $4(6.0 \%)$ \\
\hline \multicolumn{2}{|c|}{ Comorbidities } \\
\hline Anaemia & $16(23.9 \%)$ \\
\hline Malignancy & $21(31.3 \%)$ \\
\hline \multicolumn{2}{|c|}{ Electrocardiogram } \\
\hline Atrial fibrillation & $5(7.5 \%)$ \\
\hline ST depression or elevation & $4(6.0 \%)$ \\
\hline Non-specific ST changes & $6(9.0 \%)$ \\
\hline $\mathrm{T}$ wave inversion & $3(4.5 \%)$ \\
\hline
\end{tabular}




\begin{tabular}{|c|c|}
\hline \multicolumn{2}{|c|}{ Laboratory results } \\
\hline NT-proBNP (venous) $(\mathrm{pg} / \mathrm{mL})$ & $237.2 \pm 512.0$ \\
\hline NT-proBNP (capillary) $(\mathrm{pg} / \mathrm{mL})$ & $235.7 \pm 530.3$ \\
\hline Haemoglobin $(\mathrm{g} / \mathrm{L})$ & $137.5 \pm 18.0$ \\
\hline Creatinine $(\mu \mathrm{mol} / \mathrm{L})$ & $86.0 \pm 25.9$ \\
\hline Glomerular filtration rate $(\mathrm{ml} /$ & $74.1 \pm 19.2$ \\
\hline min) & $16(23.9 \%)$ \\
\hline eGFR $>90(\mathrm{ml} / \mathrm{min})$ & $37(55.2 \%)$ \\
\hline $60 \leq$ eGFR $<90(\mathrm{ml} / \mathrm{min})$ & $7(10.4 \%)$ \\
\hline $30 \leq$ eGFR $<60(\mathrm{ml} / \mathrm{min})$ & $2(3.0 \%)$ \\
\hline eGFR $<30(\mathrm{ml} / \mathrm{min})$ & $4.3 \pm 0.8$ \\
\hline Total cholesterol $(\mu \mathrm{mol} / \mathrm{L})$ & $2.1 \pm 0.7$ \\
\hline LDL cholesterol $(\mu \mathrm{mol} / \mathrm{L})$ & $1.6 \pm 0.6$ \\
\hline HDL cholesterol $(\mu \mathrm{mol} / \mathrm{L})$ & \\
\hline
\end{tabular}

67 patients with COPD were enrolled, mostly moderate $(55.2 \%)$ and severe $(35.8 \%)$ based on GOLD classification (Table $1)$. Patients were predominantly male $(68.7 \%)$, elderly $(71.5 \pm 10.0$ years), and overweight ( $66 \%$ with BMI $\geq 25$ ). Cardiovascular risk factors were common, including smoking (93\%), hypertension (37\%), dyslipidemia (40\%) and diabetes (19\%). Cardiovascular disease was also notable, including atrial fibrillation (10\%), coronary disease $(19 \%)$ and previous myocardial infarction (10\%). Anemia and mild renal impairment were common, 24\% and 55\% respectively. Moderate or severe renal disease was less frequent $(13 \%)$.

\section{Correlation}

Mean venous versus capillary values of NT-proBNP were similar, $237 \pm 512$ vs. $236 \pm 530 \mathrm{pg} / \mathrm{mL}$. The scatter-plot illustrates the linear correlation between the venous and capillary values (Figure 1), which were highly correlated with the coefficient of determination (R2) of $0.96(\mathrm{P}<0.05)$. The slope was $1.01 ; 95 \%$ CI $[0.96,1.07]$, with an intercept of $-4.91 ; 95 \%$ CI [-33.87, 24.05].

Mean \pm SD or frequency $(\%)$

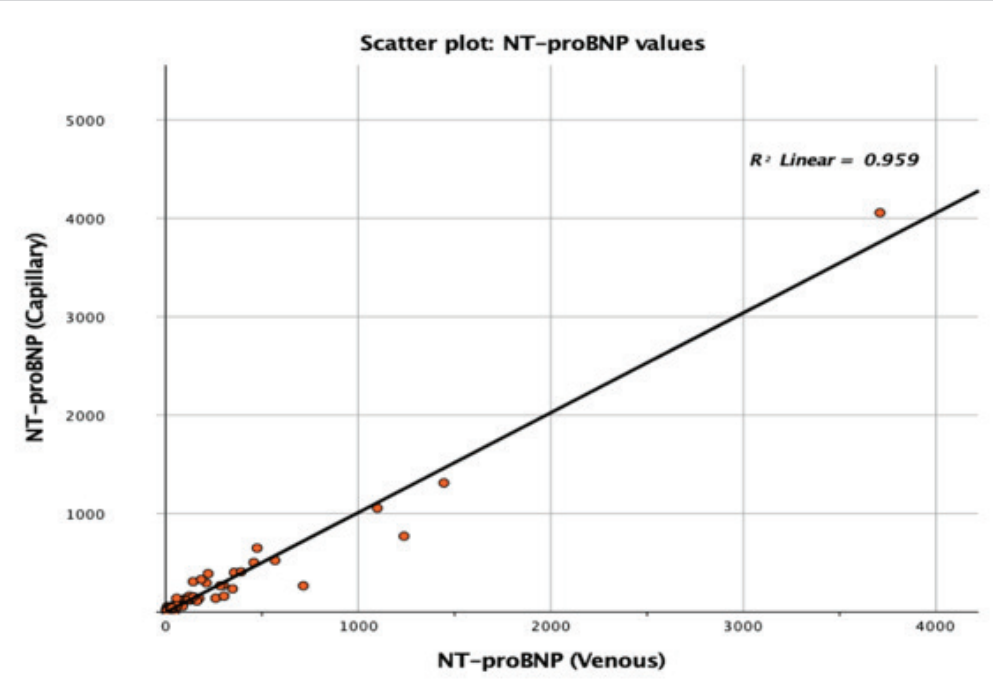

Figure 1: Scatter plot comparing venous versus capillary NT-proBNP measurements.

\section{Reclassification}

Table 2: Proportion of patients with low, medium or high NT-proBNP according to standard thresholds based on venous versus capillary measurement.

\begin{tabular}{|c|c|c|c|}
\hline Age & Low & Medium & High \\
\hline$<50$ years $(n=2)$ & $<300 \mathrm{pg} / \mathrm{mL}$ & $300-450 \mathrm{pg} / \mathrm{mL}$ & $>450 \mathrm{pg} / \mathrm{mL}$ \\
\hline Venous & 2 & 0 & 0 \\
\hline Capillary & 2 & 0 & 0 \\
\hline $50-75$ years $(n=43)$ & $<300 \mathrm{pg} / \mathrm{mL}$ & $300-900 \mathrm{pg} / \mathrm{mL}$ & $>900 \mathrm{pg} / \mathrm{mL}$ \\
\hline Venous & 26 & 15 & 2 \\
\hline Capillary & 25 & 16 & 2 \\
\hline$>75$ years $(n=22)$ & $<300 \mathrm{pg} / \mathrm{mL}$ & $300-1800 \mathrm{pg} / \mathrm{mL}$ & $>1800 \mathrm{pg} / \mathrm{mL}$ \\
\hline Venous & 11 & 11 & 0 \\
\hline Capillary & 7 & 15 & 0 \\
\hline Total & $<300 \mathrm{pg} / \mathrm{mL}$ & Medium (age-specific) & High (age-specific) \\
\hline Venous & 39 & 26 & 2 \\
\hline Capillary & 34 & 31 & 2 \\
\hline
\end{tabular}


In terms of reclassification, capillary and venous sampling was identical in identifying two middle-aged patients with probable heart failure (NT-proBNP $>900 \mathrm{pg} / \mathrm{mL}$ ) (Table 2). However, capillary testing reclassified 5 of the 39 patients (13\%) designated low probability by venous testing as having intermediate values, mainly elderly patients.

\section{Level of Agreement}

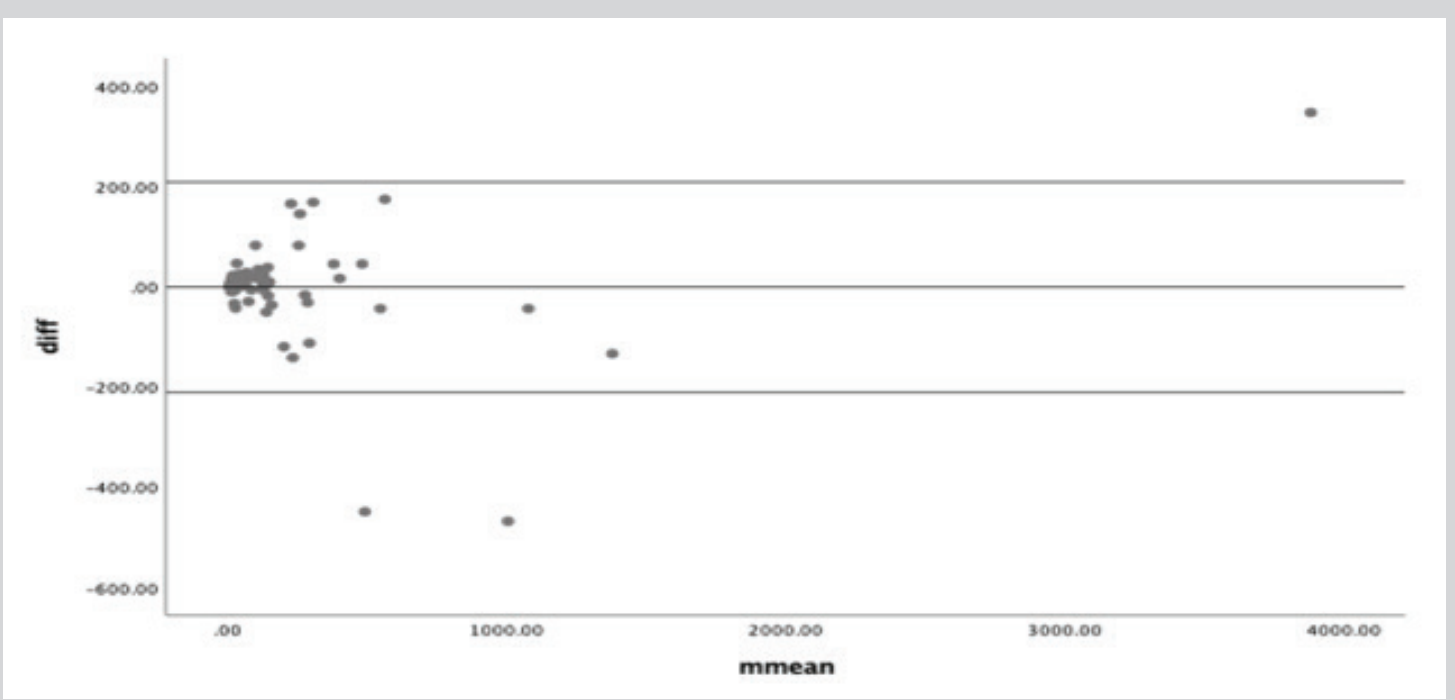

Figure 2: Bland-Altman plot.

The Bland-Altman plot visualizes the limits of agreement between venous and capillary values. It indicates that the agreement interval (-211.12 to 208.32) within which 95\% of the differences of the capillary method, compared to the venous one fall. After a linear regression analysis, there is no proportional bias pointing out that there is no trend being above or below the mean difference line.

The Bland-Altman plot (Figure 2) demonstrates the limits of agreement (-211.12 to $208.32 \mathrm{pg} / \mathrm{mL})$ in which $95 \%$ of the differences between capillary compared to venous values are expected. There was no trend above or below the mean difference line after linear regression analysis, confirming a high level of agreement.

\section{DISCUSSION}

We report several key findings in this comparison of capillary versus venous NT-proBNP measurement using a commercially available POCT assay. Overall, mean capillary and venous NTproBNP values were similar with high correlation. Detection of high NT-proBNP levels was identical. However, capillary compared to venous NT-proBNP reclassified five patients from "low" to "moderate" risk.

Relatively few studies have examined the clearance of BNP from human blood, but those that do provide a potential explanation for capillary samples' overestimating BNP [15]. There is a significant ateriovenous gradient of BNP between the femoral artery and femoral vein, implying a degree of BNP clearance in peripheral tissue [16]. This could account for higher BNP levels in the upstream capillaries compared to veins. The proposed mechanism is a combination of receptor mediated breakdown and extra-cellular proteolysis of BNP. NT-proBNP clearance is less reliant on receptor mediated breakdown and extra-cellular proteolysis. However, a significant arteriovenous gradient is still evident between the femoral artery and vein as well as across the liver, renal, head, and neck tissue [17]. This perhaps reflects the less rapid overall clearance of NT-proBNP (half-life 120 minutes vs. 20 minutes for BNP) [18].

Our study of NT-proBNP was prompted by previous studies comparing capillary and venous BNP. In a cohort of 111 patients with stable HF, overall capillary and venous BNP levels correlated strongly $(\mathrm{r}=0.94)$. However, this diverged at BNP levels greater than $1500 \mathrm{pg} / \mathrm{mL}$, at which point capillary testing tended to overestimate BNP with a bias of $46.9 \mathrm{pg} / \mathrm{mL}$ seen on Bland-Altman analysis [8]. A similar correlation between capillary and venous BNP levels was observed in 98 healthy adult subjects and patients diagnosed with systolic HF in another single centre study ( $\mathrm{r}=0.96)$. There was a non-significant trend towards bias with the capillary method overestimating BNP by $14.9+/-40.5 \%$ ( $\mathrm{p}=0.04$ ) [19]. Finally, in a similar sized, single-centre study ( $\mathrm{n}=117)$, capillary was consistently higher than venous BNP, with a regression slope (i.e. correction factor) of 1.48. Limited information was provided in the study, though such differences may typically be attributable to differences in sample handling, sample anticoagulation, and assay platforms [20].

Overall, the strong correlation between capillary and venous NT-proBNP values in our study suggests there may be a role for capillary NT-proBNP POCT for diagnosis and monitoring of HF. In five instances, capillary blood samples generated higher NT-proBNP readings than venous samples, resulting in a reclassification from low to moderate BNP levels. Potentially higher capillary values could lead to false positive diagnoses of HF and thus decrease the positive predictive value. This would require increased use of confirmatory imaging, though not necessarily impact the negative predictive value and utility as a screening tool. Further validation studies are needed to assess whether higher cut-off values for HF diagnosis or a correction factor, as seen in prior studies, are needed for capillary NT-proBNP measurements [20].

A finger prick blood sample is collected via pipette, mixed 10 times with a buffer solution, and then applied to a single-use 
disposable cartridge to use the RAMP $₫$ kit and reader. This process introduces opportunities for blood clotting and other errors. A more streamlined process in which blood from a finger prick is applied directly to a cartridge would be preferable. This exists for BNP testing and was shown to be feasible for home monitoring in the HABIT study [21]. To our knowledge this is not currently available for NT-proBNP testing.

There are several limitations to our study. The sample size of 67 is relatively small. The COPD population limits the generalizability to other target populations, such as patients presenting with unexplained dyspnea. However, similar results were seen in both stable HF patients and healthy adult volunteers using BNP $(8,19,20)$. The lack of imaging limits our ability to determine the accuracy and predictive values of capillary NT-proBNP POCT as compared to the gold standard for the diagnosis of HF.

Further research is required to clarify the role of capillary NTproBNP POCT for the diagnosis and management of HF. Larger sample sizes, including more patients with elevated NP levels and $\mathrm{HF}$, are needed to clarify the reclassification at the upper threshold for probable HF. Including a gold standard would allow assessment of the accuracy of the parameters and recalibration of diagnostic thresholds if necessary. Nonetheless, this is an encouraging first step in the incorporation of NT-proBNP POCT.

\section{CONCLUSION}

There is a good correlation between NT-proBNP levels in capillary and venous samples, which suggests there may be a role for NT-proBNP POCT using capillary blood samples. These findings need to be confirmed in larger and more diverse populations, including more patients with HF.

\section{REFERENCES}

1. Ezekowitz JA, O’Meara E, McDonald MA, Abrams H, Chan M, et al. (2017) 2017 Comprehensive update of the Canadian Cardiovascular society guidelines for the management of heart failure. Can J Cardiol 33(11): 1342-433.

2. Taylor KS, Verbakel JY, Feakins BG, Price CP, Perera R, et al. (2018) Diagnostic accuracy of point-of-care natriuretic peptide testing for chronic heart failure in ambulatory care: Systematic review and metaanalysis. BMJ 361.

3. Morbach C, Buck T, Rost C, Peter S, Günther S, et al. (2018) Point-ofcare B-type natriuretic peptide and portable echocardiography for assessment of patients with suspected heart failure in primary care: rationale and design of the three-part Handheld-BNP program and results of the training study. Clin Res Cardiol 107(2): 95-107.

4. Monfort A, Silva K Da, Vodovar N, Gayat E, Cohen-Solal A, et al. (2015). Clinical evaluation of the heart check system, a new quantitative measurement of fresh capillary BNP. Biomark Med 9(12): 1323-1230.

5. Ponikowski P, Voors AA, Anker SD, Bueno H, Cleland JGF (2016) 2016
ESC Guidelines for the diagnosis and treatment of acute and chronic heart failure. Chinese J Biol 24(11): 1249-1253.

6. Nayer J, Aggarwal P, Galwankar S (2014) Utility of point-of-care testing of natriuretic peptides (brain natriuretic peptide and n-terminal probrain natriuretic peptide) in the emergency department. Int J Crit Illn Inj Sci 4(3): 209-215.

7. Pan Y, Li D, Shan L, Wei M (2017) NT-proBNP test with improved accuracy for the diagnosis of chronic heart failure. Medicine 96(51): e9181.

8. De Vecchis R, Ariano C (2016) Measuring B-type natriuretic peptide from capillary blood or venous sample: Is it the same? Cardiol Res 7(2): 51-58.

9. Inçaurgarat B, Nieuwenhuis J (2012) Next-generation, fast and accurate point-of-care test for NT-proBNP based on Magnotech technology. Crit Care16(1): P184.

10. Point of care precision and sample comparison study of minicare BNP at innsbruck hospital - MI-BNP-CE04-AN2016.

11. Detect cancer and prevent heart failure-with a simple prick of the finger, Pressat.

12. Johannes MM (2016) Clinical utility of BNP for monitoring patients with chronic HF treated with sacubitril-valsartan. JACC 2016: 1-9.

13. Piek A, Du W, de Boer RA, Silljé HHW (2018) Novel heart failure biomarkers: why do we fail to exploit their potential? Crit Rev Clin Lab Sci 55(4): 246-263.

14. Januzzi JL, van Kimmenade R, Lainchbury J, Bayes-Genis A, OrdonezLlanos J, et al. (2006) NT-proBNP testing for diagnosis and short-term prognosis in acute destabilized heart failure: an international pooled analysis of 1256 patients: The International Collaborative of NT-proBNP Study. Eur Heart J 27: 330-337.

15. Potter LR (2011) Natriuretic peptide metabolism, clearance and degradation. FEBS J 278(11): 1808-1817

16. Richards AM, Crozier IG, Yandle TG, Espiner E, Ikram H (1993) Brain natriuretic factor: regional plasma concentrations and correlations with haemodynamic state in cardiac disease. Br Heart J 69(5): 414-417.

17. Palmer SC, Yandle TG, Nicholls MG, Frampton CM, Richards AM (2009) Regional clearance of amino-terminal pro-brain natriuretic peptide from human plasma. Eur J Heart Fail 11(9): 832-839.

18. Kim HN, Januzzi JL (2011) Natriuretic peptide testing in heart failure. Circulation 123(18): 2015-2019.

19. Prontera C, Masotti S, Franzini M, Emdin M, Passino C, et al. (2015) Comparison between BNP values measured in capillary blood samples with a POCT method and those measured in plasma venous samples with an automated platform. Clin Chem Lab Med 53(5): 125-127.

20. Reenen AV, Berger M, Moreau E, Bekx E, Bruinink T, et al. (2019) Analytical performance of a single epitope B-type natriuretic peptide sandwich immunoassay on the minicare platform for point-of-care diagnostics. Pract Lab Med 15: e00119.

21. Maisel A, Barnard D, Jaski B, Frivold G, Marais J, et al. (2013) Primary results of the HABIT trial (heart failure assessment with BNP in the home). JACC 61(16): 1726-1735. 